\title{
The role of MR volumetry in brain atrophy assessment in multiple sclerosis: A review of the literature
}

\author{
Ewelina Marciniewicz ${ }^{A-D}$, Przemysław Podgórski ${ }^{B-D}$, Marek Sąsiadek ${ }^{E, F}$, Joanna Bladowska ${ }^{A-F}$ \\ Department of General Radiology, Interventional Radiology and Neuroradiology, Wroclaw Medical University, Poland \\ A - research concept and design; B - collection and/or assembly of data; C - data analysis and interpretation; \\ $D$ - writing the article; $E$ - critical revision of the article; $F$ - final approval of the article
}

Address for correspondence

Ewelina Marciniewicz

E-mail: ewelina.marciniewicz@gmail.com

Funding sources

None declared

Conflict of interest

None declared

Received on February 13, 2018

Reviewed on May 7, 2018

Accepted on August 9, 2018

Published online on February 6, 2019

Cite as

Marciniewicz E, Podgórski P, Sąsiadek M, Bladowska J.

The role of MR volumetry in brain atrophy assessment in

multiple sclerosis: A review of the literature. Adv Clin Exp Med.

2019;28(7):989-999. doi: 10.17219/acem/94137

DOI

10.17219/acem/94137

\section{Copyright}

Copyright by Author(s)

This is an article distributed under the terms of the

Creative Commons Attribution Non-Commercial License

(http://creativecommons.org/licenses/by-nc-nd/4.0/)

\begin{abstract}
We review the current role of magnetic resonance (MR) volumetry as a meaningful indicator of neurodegeneration and clinical disease progression in multiple sclerosis (MS) patients. Based on a review of the current literature we summarize the mechanisms that contribute to brain atrophy. We present the newest magnetic resonance imaging (MRI)-based methods used in atrophy quantification. We also analyze important biological factors which can influence the accuracy of brain atrophy evaluation. Evidence shows that measures of brain volume (BV) have the potential to be an important determinant of disease progression to a greater extent than conventional lesion assessment. Finally, scientific reports concerning limitations of MRI-based volumetry that affect its implementation into routine clinical practice are also reviewed. The technical challenges that need to be overcome include creating a standardized protocol for image acquisition - a fully automated, accurate and reproducible method that allows comparison in either single-center or multicenter settings. In the near future, quantitative MR research will probably be the basic method used in neurology to monitor the rate of atrophic processes and clinical deterioration in MS patients, and to evaluate the results of treatment.
\end{abstract}

Key words: magnetic resonance imaging, multiple sclerosis (MS), brain atrophy, MR volumetry 
Multiple sclerosis (MS) is a chronic disabling disease. It is one of the earliest-known neurological diseases, described by Charcot in 1868. It is a progressive neurological disorder resulting in both physical and neurocognitive disability. ${ }^{1}$ In the US, there are approx. 400,000 people currently diagnosed with MS but it is estimated that around 2.5 million people worldwide might suffer from it. The disease typically begins in early adulthood with variable prognoses; $50 \%$ of the patients will require aid in walking within 15 years. The process of diagnosing and understanding MS involves specialists from neurology, immunology, radiology, and many other disciplines. ${ }^{2}$ In MS, the brain is attacked and destroyed by the body's own immune system, causing physical disability and cognitive impairment. ${ }^{3}$ This results in cerebral changes that lead to brain atrophy at a much higher rate than when this process occurs with aging in healthy people. ${ }^{4}$

Magnetic resonance imaging (MRI) is a very useful tool not only in the process of initial diagnosis of MS, but also in monitoring disease progression and therapeutic response. Current MRI volumetric techniques can detect brain volume changes with an accuracy of $3 \mathrm{~mL}$. This precise quantitative information has significant potential for evaluating disease activity and changes following therapy. ${ }^{5-7}$ Magnetic resonance imaging volumetry became a clinically relevant component of disease assessment because of its high sensitivity and specificity in detecting volumetric changes of the brain. ${ }^{8}$ It allows whole-brain volume to be measured, as well as the volume of particular brain lobes and gyri. ${ }^{9}$ In general, whole brain atrophy is considered a good predictor of long-term clinical disability in all stages of MS. ${ }^{10}$ Nevertheless, it should be always combined with assessments of the clinical state of a patient.

Within the last 10 years, numerous studies conducted on MS patients have reported accelerated brain atrophy rate in comparison with healthy subjects. ${ }^{11,12}$ Hardmeier et al. ${ }^{11}$ documented that gray matter (GM) volumes are lower in MS patients than in healthy age-matched controls. In addition, annual brain tissue loss occurred at a faster rate in MS patients $(0.5-1 \%$ per year) than in the control group (0.1-0.3\% per year). Global brain atrophy has been demonstrated in all subtypes of MS: in relapsingremitting (RR), secondary progressive (SP) and primary progressive (PP) MS. ${ }^{2,13,14}$ A considerable amount of data confirms that measurements of the percentage of brain tissue loss over time are among the best methods for quantifying neurodegeneration in MS and monitoring disease progression. ${ }^{6}$

In this paper, we review the data regarding MRI volumetric techniques and their clinical applications. We focus on the relationships between the severity of clinical symptoms, including disability, and the percentage of brain atrophy. We also present prospects for future uses of MRI metrics in the process of diagnosing and assessing brain volume (BV) changes in the course of MS.

\section{The pathomorphological basis of atrophy in multiple sclerosis}

Atrophy is seen at all clinical stages of MS and develops gradually. The loss of brain tissue is mainly due to myelin loss. However, there are also other tissue elements that contribute to whole BV loss, such as changes in tissue water content and the loss of glial cells, vascular elements and GM.

Furthermore, factors like protein catabolism, changes in electrolyte balance, vascular permeability, and dehydration may have an influence on brain atrophy. Changes caused by the inflammation process may also play a role. The main target for researchers has been to determine the pathologic process that leads to the development of plaques in MS. A lack of integrity in the blood-brain barrier is a mechanism that has received a great deal of attention; it allows the entrance of leukocytes into the normally immunologically privileged central nervous system (CNS) as a response to inflammation. The myelin sheath located in the CNS becomes the target of immunologic attack. This underlying pathology is present from the beginning of the disease, sometimes long before clinical symptoms are present, and continually progresses. The neurodegeneration translates to macroscopic brain atrophy that can be quantified in vivo through brain MRI. ${ }^{8}$

Multiple sclerosis has long been classified as a primary white matter (WM) disease of the CNS. Foci of demyelination in WM are readily visualized using MRI and remain a hallmark of the disease. Pathologic studies report that GM is also affected, and seems to be a large component of all the pathologies caused by MS. This damage includes widespread demyelination, neuron apoptosis, and atrophy affecting the cortex and deep GM structures. ${ }^{9}$

Nevertheless, inflammation also plays a protective role by expressing growth factors that promote remyelination and protect against axonal damage. ${ }^{10}$ These neurotrophins are essential for normal oligodendrocyte development: they promote migration, differentiation and maturation of oligodendrocyte precursor cells. ${ }^{11}$ These beneficial effects of inflammation have become an extremely promising therapeutic approach to MS.

\section{The concept of neurodegeneration in multiple sclerosis}

Although demyelination with hyperintense lesions on T2-weighted MRI remains the main diagnostic criterion of MS, there is growing evidence that the extent of clinical disability does not correlate with the number of foci. It has been reported that the extent of total axonal damage resembling neurodegeneration shows a strong correlation with clinical progression and strongly predicts irreversible disability. ${ }^{8,12,15,16}$ 
Reductions in N-actetylaspartate (NAA, a marker of axonal integrity) have also been observed in magnetic resonance spectroscopy (MRS) at a very early phase of the disease. ${ }^{17}$ This finding indicates the neurodegenerative component of the MS background. A better understanding of the molecular mechanisms underlying MS progression will aid the development of new treatment strategies based on both anti-inflammatory and neuroprotective action.

\section{Insight into atrophy measurements in multiple sclerosis}

Conventional MRI techniques, using markers such as T2-weighted lesions and gadolinium-enhancing lesions, are now widely used in clinical practice in the diagnosis and follow-up of MS. However, the utility of these MRI measures in predicting disease progression in MS is limited. New areas of gadolinium enhancement are considered good markers for clinical relapses. Nevertheless, clinical deterioration is not clearly related to inflammatory lesions, but rather to a progressive and diffuse neuroatrophy. ${ }^{18}$

Recent evidence shows that axonal loss and dysfunction occur very early in the course of the disease and are apparent at all stages of the disease. ${ }^{17}$ Atrophy is seen not only within the lesions but also in normal-appearing WM, where it is probably secondary to myelin loss and axonal damage caused by Wallerian degeneration. ${ }^{8}$ For that reason in the past decade brain atrophy has become one of the most important indicators of neurodegeneration and clinical disease progression in MS patients. This probably reflects both inflammation-induced axonal loss and post-inflammatory neurodegeneration that may be due to inefficient remyelination. ${ }^{19,20}$ Moreover, it has been shown that atrophy measures correlate with disability indices and cognitive performance in MS patients. ${ }^{21,22}$

Diffuse tissue loss in normal-appearing brain tissue can be monitored in a sensitive and reproducible manner using quantitative MR measurement. Magnetic resonance techniques provide an objective and direct assessment of the evolving pathology in MS. ${ }^{8}$ This is a point of interest for trials of new agents aimed at preventing disability. Atrophy measurements should be included as an endpoint in trials of all disease-modifying agents to monitor treatment efficacy.

\section{Factors affecting brain volume changes over time in multiple sclerosis}

Many studies have demonstrated that there is no direct correlation between BV changes and patient's clinical state and rate of disability. ${ }^{8,23}$ This suggests that there are other factors, unrelated to the disease, that influence BV changes in MS patients. This requires further investigation. For example, any inflammatory reaction can temporarily increase $\mathrm{BV}$, causing vasogenic edema. In addition, processes like glial cell proliferation and gliosis can also contribute to false BV increase. ${ }^{24}$

There are other biological factors, including body fluid status, nutrition, addictions, menstrual cycle, genetic and environmental considerations, and gender- and agerelated features which may contribute to BV changes. These can all cause physiologic variations in BV. Cerebral volume loss progresses with age but may be more pronounced when there are other risk factors such as alcohol abuse, smoking, dehydration, or concomitant diseases (e.g., diabetes, cardiovascular risk factors or Cushing's syndrome). All this may affect the accuracy of BV measurements. ${ }^{25,26}$

Duning et al. ${ }^{27}$ showed that hydration status can significantly change BV: a lack of fluid intake for $16 \mathrm{~h}$ decreased BV by $0.55 \%$ (standard deviation $(\mathrm{SD})=0.69$ ) and after rehydration total BV increased by $0.72 \%(\mathrm{SD}=0.21$ ). Changes as high as 30-40 mL have also been observed after dialysis in patients with renal failure. ${ }^{28}$ Dehydration can confound the assessment of brain atrophy.

For these reasons, in cases in which small changes of total BV are important diagnostic parameters - for example in MS and neurodegenerative diseases - comparative hydration status should be considered in longitudinal automated MR-based measurements of BV. ${ }^{29}$

Another important factor which should be considered is weight loss. Some studies on patients with anorexia nervosa (AN) have already revealed various effects of nutritional status on BV. Enlargement of the cortical sulci and cerebrospinal fluid spaces as well as pituitary gland atrophy in patients with AN have been reported. ${ }^{25}$ Heinz et al. postulated that the mechanism responsible for the atrophic changes may be related to protein loss and fluid retention caused by hypercortisolism and loss of serum protein. This suggests that there is no simple correlation between cerebral atrophy and malnutrition. It should be emphasized that although in some cases of AN morphological brain changes have been reported, we cannot consider BV dependence on the nutritional state of the patient. ${ }^{30,31}$

It should be taken into consideration that the correlation between brain loss and progression of the disease is influenced by a phenomenon called brain plasticity. In patients with MS it is believed to be a compensatory mechanism based on activation of new brain areas in order to retain some brain functions. Neuroplasticity is a property of the nervous system to obtain functional goals. The increased functional recruitment of the cortex in MS patients might have an adaptive role in limiting the clinical impact of irreversible tissue damage. Cortical motor reorganization has been described in various diseases including tumors, vascular malformations and stroke. ${ }^{32}$ 
There is a growing body of evidence suggesting that functional cortical changes develop early in the disease and have a role in limiting the clinical impact of MS injury. Therefore, MS-related brain damage may occur undetected during the early phase of the disease. Moreover, MS may remain undiagnosed and untreated for a long time. ${ }^{33}$ As adaptive cortical changes have the potential to limit the clinical impact of MS injury, the question arises of how strong the correlation is between MR volumetry and clinical outcomes in MS patients. This interrelation is still to be explored. ${ }^{34}$

The clinical value of brain atrophy measurements in MS necessitates future longitudinal follow-up studies conducted in a standardized manner on a large group of patients. Brain volume changes should be measured over at least a 1-year period and compared with changes in disability measured using disability status scales. The pathophysiological significance of altered brain activation patterns in MS patients and their influence on the clinical state of patients also needs further exploration.

There is also a strong need for validation of brain atrophy rates through longer-lasting trials in order to establish stable baselines. This will enable better interpretation of BV changes over time and allow introducing quantitive BV assesment into routine clinical practice.

\section{The phenomenon of pseudoatrophy}

Pseudoatrophy is a temporary phenomenon that represents accelerated water loss and reduced edema during the course of anti-inflammatory treatment. It is not associated with neuron or tissue damage. The mechanisms responsible for this effect include the induction of protein catabolism and the reduction of water volume in the brain as a result of decreased vascular permeability without real axonal loss.

Disease-modifying drugs at treatment initiation reduce edema, inflammation and extracellular water in the brain, leading to pseudoatrophy. It may initially confound real treatement effects in the first few months of therapy. ${ }^{35}$ Many volumetric studies confirmed that changes in the volume of inflammatory cells, particularly glial cells, may be also relevant. ${ }^{36}$ Several independent studies have reported that anti-inflammatory drugs transiently decrease BV within the first 6 months to 1 year of treatment. ${ }^{11,37}$ An acute effect of intravenously administrated corticosteroids on BV has been shown by several researchers. ${ }^{38-40}$ Hardmeier et al. ${ }^{11}$ explored the kinetics of atrophy after treatment initiation. Their study included 802 patients with active RR MS disease. Among them, 189 subjects were randomized to $30 \mu \mathrm{g}$ and 197 subjects to $60 \mu \mathrm{g}$ of interferon beta-1a (IFN-1a IM) once a week. Brain parenchymal fraction (BPF), a normalized measure of whole-brain atrophy, and the volume of Gd-enhancing lesions (T1Gd) and T2 hyperintense lesions (T2LL) were evaluated. The BPF analysis showed a decrease of BV in the first year of treatment. Nearly $70 \%$ of BPF change occurred during the first 4 months and was accompanied by a drop in T1Gd volume. This nonlinear development of atrophy in the first phase of treatment is caused by edema resolution and the effect of IFN-1a on the blood-brain barrier, both imitates atrophy and can cause difficulties in assessing disease progression and the efficacy of immunomodulating and immunosuppressive therapies. The authors suggest that yearly measurement of BV loss after therapy may not be optimal for assessing atrophy changes. They have highlighted the importance of obtaining a "second baseline" after 4 or 6 months of treatment.

The same mechanism is also observed in patients with non-neurologic autoimmune diseases, for example Cushing's disease and other autoimmune diseases treated with prolonged daily use of corticosteroids over the midto long-term and showing significant BV loss. ${ }^{31,41,42}$

Another novel finding, presented in a study by Filippi et al., was the percentage of BV change (PBVC). The mean PBVC for patients on a placebo was $-0.83 \%$ during the $1^{\text {st }}$ year, $-0.67 \%$ during the $2^{\text {nd }}$ year and $-1.68 \%$ during the entire study period. The respective values for treated MS patients were $-0.62 \%,-0.61 \%$ and $-1.18 \%$. The explanation for these findings might be the ability of the treatment to reduce Gd-enhancing lesions and edema during the first phase of treatment, which in turn might result in pseudoatrophy. ${ }^{43}$

In contrast, focal edema in new demyelinating lesions may compensate true BV loss from tissue destruction, especially in WM, where inflammation is more pronounced. On the other hand, the process of remyelination within the lesion may mask atrophy. ${ }^{29}$ This fact has important implications for the design of clinical trials. It is necessary to further investigate how much the pseudoatrophy in MS brains after the initiation of certain therapies may be related to the reduction of inflammation in order to distinguish it from true neurodegeneration.

Dynamic changes in BV in MS patients are therefore a combination of destructive and rebuilding processes of inflammation, neurodegeneration and correct, and the influence of immunomodulatory therapy. Therefore, it is very important to be aware of all these factors when interpreting whole-brain volume data because reductions in whole-brain volume may be associated not only with true atrophy. ${ }^{44}$

There is a strong need to establish normative values for BV changes - both for healthy subjects and for patients with MS - that take into account these confounding factors. These studies will enable the reliable use of whole BV measurements to prognose and predict therapeutic response.

\section{Gray matter atrophy in multiple sclerosis}

Recent studies report that clinical disability in MS patients cannot be explained by changes in WM observed in conventional MRI sequences. This suggests that GM damage 
and subsequent GM atrophy can be relevant to the development of disability. ${ }^{45,46}$ Indeed, cortical and hippocampal lesions result in symptoms of cognitive and processing speed impairments. ${ }^{47-49}$ The detection of GM alterations will play an important role in the understanding of both physical and neurocognitive disability observed in MS patients. Measurements of WM and GM atrophy remain among the current methods that can be used to quantify the grade of disease progression. ${ }^{46}$

There have been a number of studies that investigated which brain structures are affected by atrophy in different MS subtypes and how early this pathology begins. Scientists have also focused on determining which cortical areas are primarily affected in patients presenting with their first clinically isolated syndrome (CIS). Several cross-sectional studies have reported that diffuse cerebral atrophy in MS is not sensitive enough in case of first episode of neurologic symptoms. This indicates a need for a more accurate biomarker for patients after the CIS as well as those with a short disease duration. ${ }^{50}$

A number of histopathological and MRI studies have demonstrated that significant global cortical thinning is a diffuse and early phenomenon in MS. ${ }^{4,51,52}$ It correlates with clinical disability and is partially independent from WM inflammatory pathology. A longitudinal study carried out by Dalton et al. ${ }^{53}$ in patients with CIS found progressive cortical atrophy in those subjects who developed a confirmed diagnosis of MS within 3 years of the clinical onset of CIS. They reported that the mean decrease in GM fractional (GMF) volume was $-3.3 \%$ while in CIS subjects who had not developed MS it was $-1.1 \%$. No decrease in WM fractional (WMF) volume was seen. Changes in GMF volume correlated only modestly with changes in the T2 lesion load. These results suggest that progressive GM atrophy is present in the earliest clinically observable stages of relapse-onset MS, and this is only moderately related to lesion accumulation.

Recent data based on a large cohort of patients has proven that cortical thinning is already present and diffuse in a very early MS phase, when WM damage appears to be only modest. Moreover, cortical thinning is correlated with clinical disability and cognitive impairment more strongly than other MRI measurements. ${ }^{54,55}$ Among many software techniques that enable the in vivo MRIderived quantitative measurement of cerebral cortex thickness, one of the most often used is FreeSurfer (Laboratory for Computational Neuroimaging, Charleston, USA; Fig. 1). These measurements provide novel insights into neuroanatomical abnormalities during the course of the disease. Gray matter appears to be less sensitive to pseudoatrophy than WM, and could be a potentially attractive additional measurement for neuroprotection trials. ${ }^{24,51,56}$

Calabrese et al. ${ }^{57}$ carried out a 5 -year prospective longitudinal study in order to evaluate the extent to which cortical lesion load is associated with longer-term physical and cognitive disability. Patients with high cortical lesion loads had higher Expanded Disability Status Scale increases $($ median $=1.5$; range $=0-3$ ) and showed a greater GM fractional loss during the study than both patients with low cortical lesion loads and without cortical lesions. Analyzing cortical pathology may help in the early identification of patients with a worse prognosis and provide relevant clinical and therapeutic implications. Nevertheless, the extent to which MRI parameters of cortical thinning may be used for clinical purposes needs to be confirmed in further prospective longitudinal studies.

Considerable evidence exists to support the hypothesis that there are also another GM structures which seem to be more predictive than others. According to many studies, out of all subcortical GM areas, the thalamus is the structure most vulnerable to atrophy. Thalamic tissue volume loss has been found in all MS subtypes. ${ }^{58}$ Thalamic nuclei are GM structures that play a major role in cortical activation, relaying sensory information to the higher cortical centers that influence cognition, sensory and motor functions. Involvement of these structures is associated with a wide range of clinical manifestations, including cognitive impairments and motor deficits in patients with MS. ${ }^{59}$ It might become an important biomarker of disease progression.

To summarize, thalamic atrophy and cortical thickness are 2 very promising GM MRI metrics. Still more studies and longitudinal follow-ups are needed to characterize atrophy progression over time and the clinical relevance at both the group and the individual level.

\section{Overview of quantitative volumetric MRI techniques}

Numerous methods are available for the measurement of global and regional BV. ${ }^{60}$ The optimal method for measuring brain tissue loss must be sensitive to small changes, reproducible and stable over time. Techniques of MRI analysis, measuring the size of brain structures, have ranged from manual traces for estimates of diameters or sectional areas of structures to fully automated methods based on advanced computational algorithms (Fig. 2).

It is important to note that a wide variety of techniques available for measuring tissue damage are not directly comparable with each other, which makes it very difficult to compare the results of different studies based on different tools.

Although automated voxel-based methods are designed to be robust and reliable structural change detection methods, it is known that they can be influenced by physical, methodological and physiological factors such as the scan parameters, the type of coil, MRI scanner field strength, the type of applied pulse sequence, the level of patient's hydration, the phase of women's menstrual cycle, and other factors. The potential impact of the scanning parameters can be minimized with strict adherence to a specific MRI scanner and imaging protocol. 


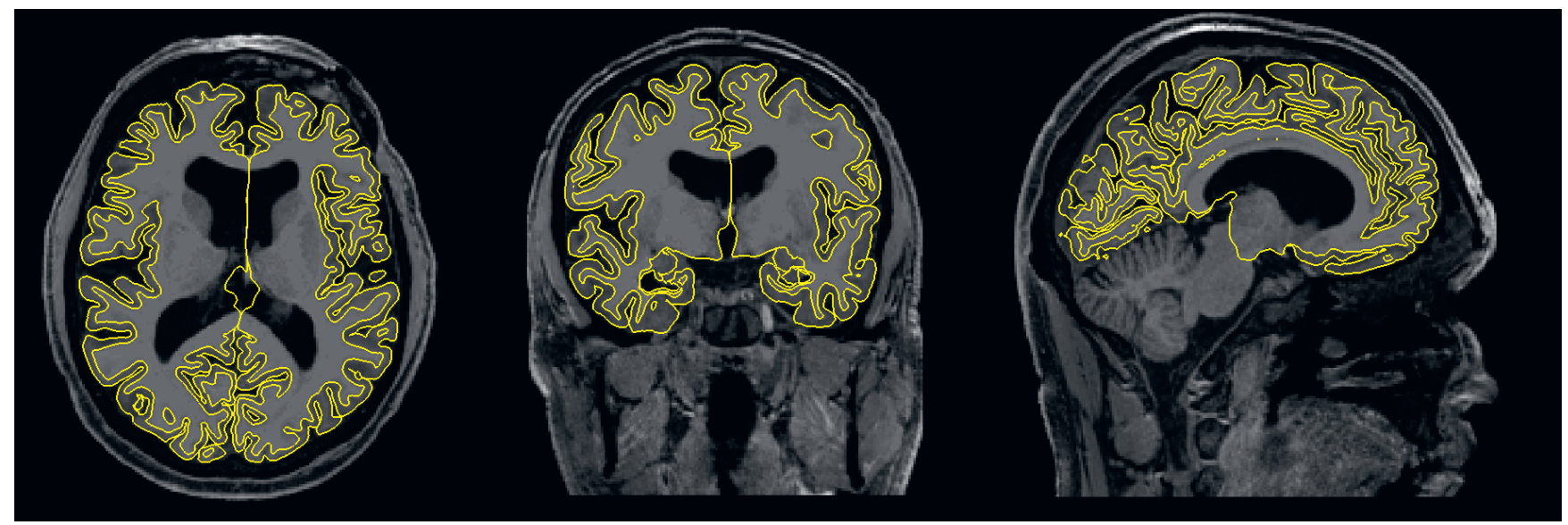

Fig. 1. Cortical thickness borders overlaid on an anatomical T1-weighted Freesurfer image

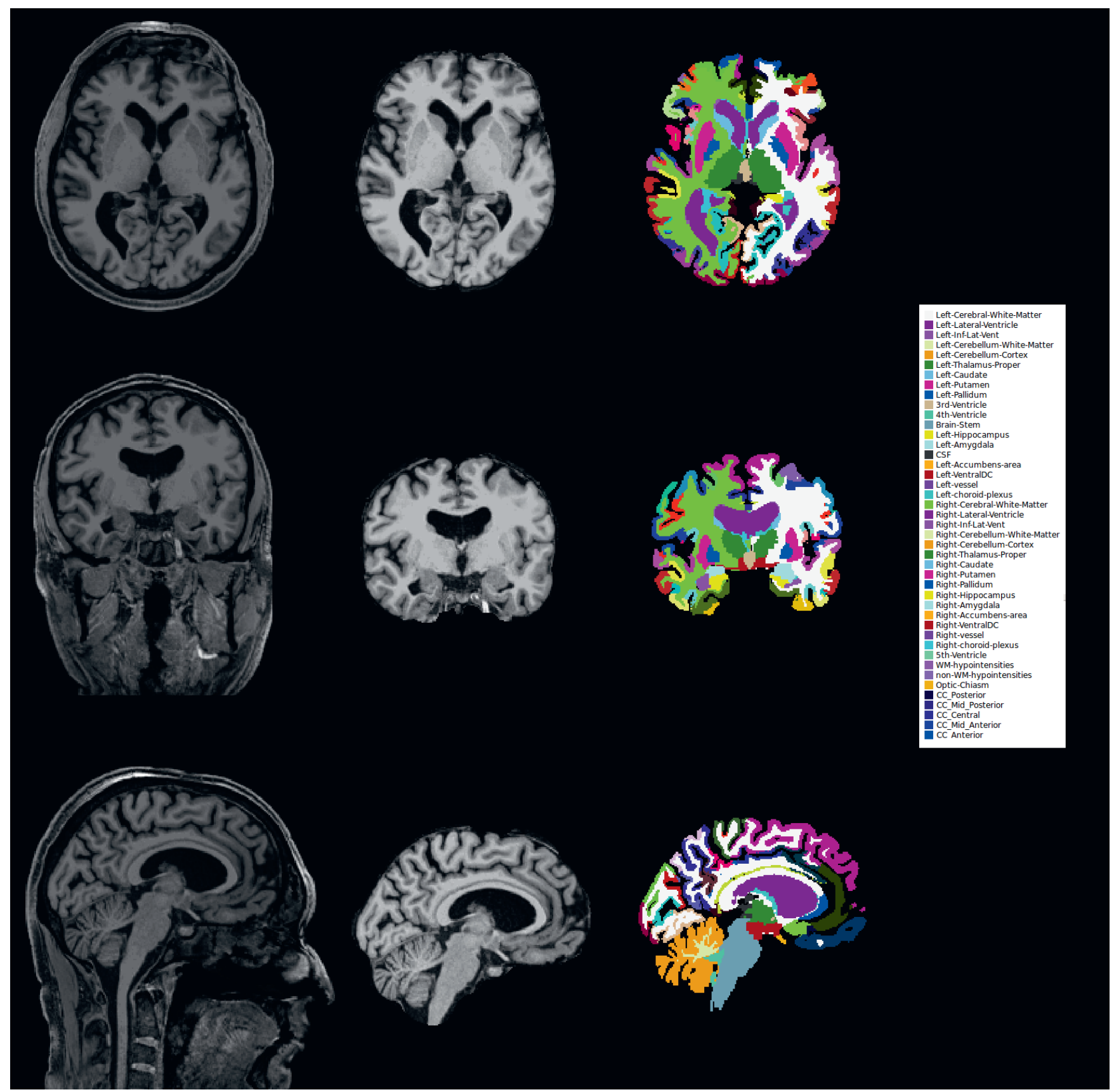

Fig. 2. Example of Brainsuite processing steps for volumetric assessment 


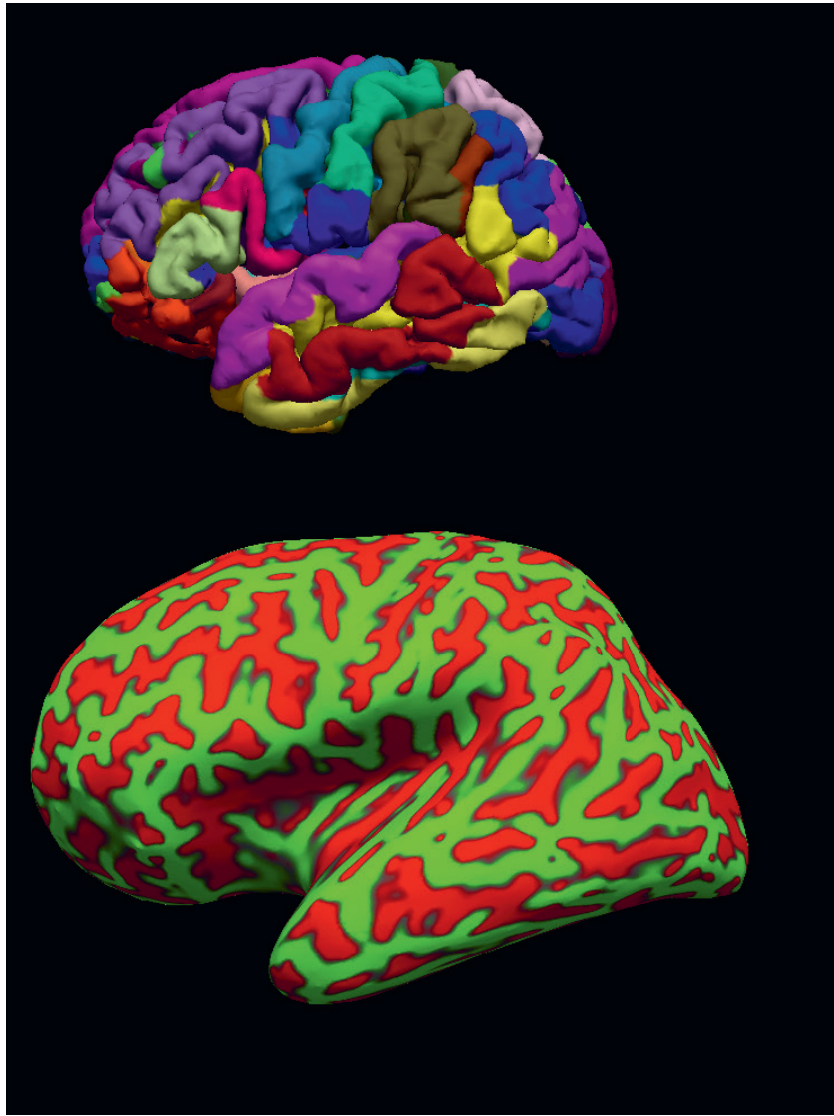

Fig. 3. Example of brain cortical and subcortical labeling using Freesurfer
Manual methods of volumetry are time-consuming and require great expertise in anatomy. The most common manual methods are the bicaudate ratio, brain width, the corpus callosum area, the midbrain to pons ratio, the lateral width, and the third ventricle width. ${ }^{61}$

A strong need for more objective, comparable and reproducible assessment of BV led to the creation of a variety of fully-automated tools such as SPM/VBM - Statistical Parametric Mapping (www.fil.ion.ucl.ac.uk/spm), Freesurfer, SIENAX, SIENA (Structural Image Evaluation using Normalisation, of Atrophy), FSL (FMRIB Software Library, Analysis Group, Oxford, UK), and BrainVISA (brainvisa.info). ${ }^{62}$

Among existing medical image segmentation techniques, one of the most popular is SIENA software, which allows measurement of whole and partial BV. The reproducibility of the measurements obtained is about $0.5 \%$. To perform more specific volume measurements, one can use segmentation-based techniques that have the potential to automatically segment subcortical brain structures and can separately quantify the volume of GM and WM, as well as the volumes of particular gyri and lobes. ${ }^{63}$ (Fig. 3). The performance of such methods depends on image registration accuracy and anatomical differences between the study subject and the atlas images (Fig. 4). This is why multi-atlas-based methods for subcortical structure segmentation have attracted a great deal of attention, partially because the spatial positions of anatomical structures are relatively stable among patients. ${ }^{64}$

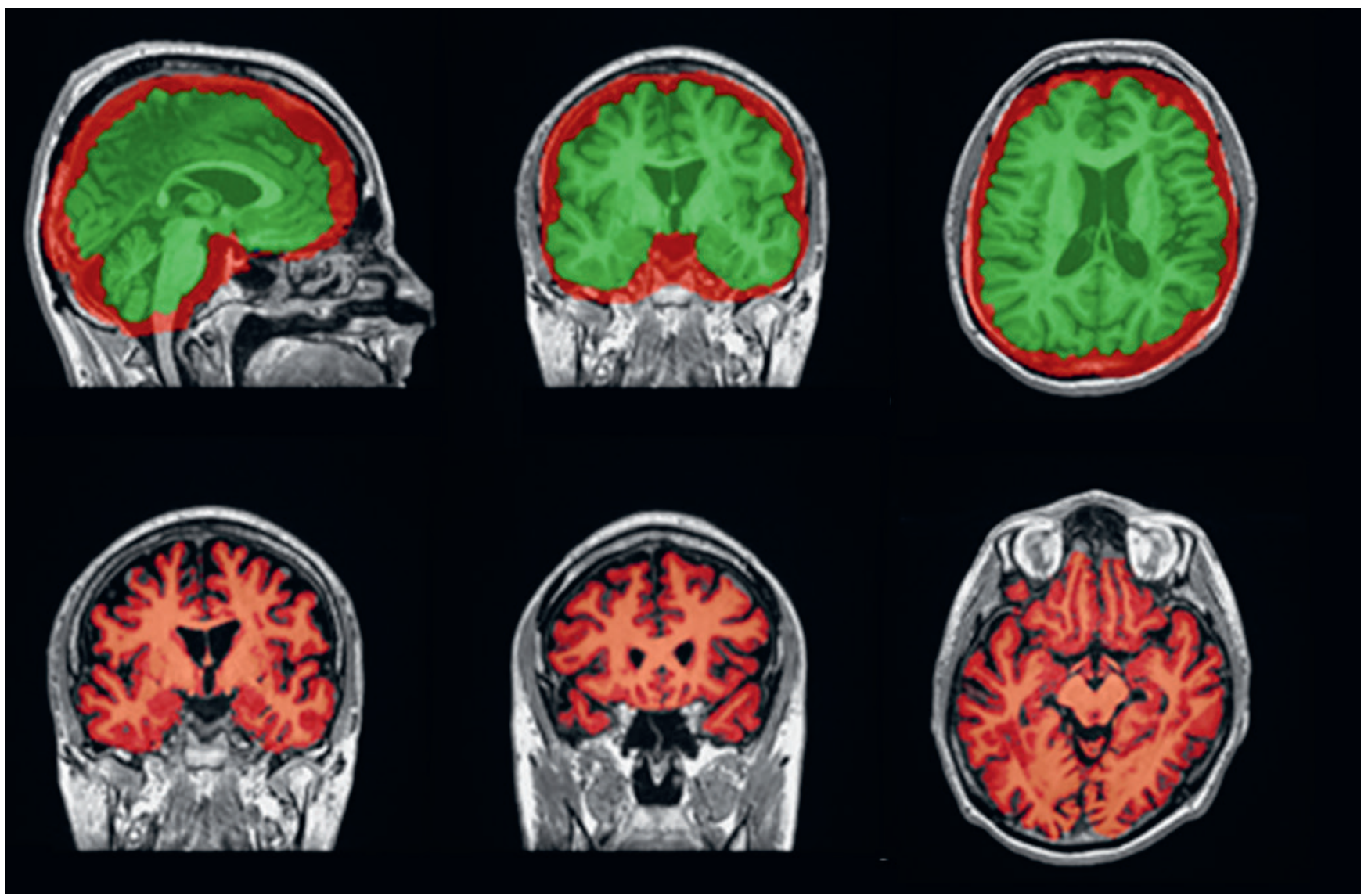

Fig. 4. Cortical thickness map (BrainVoyager QX cross-platform software) 

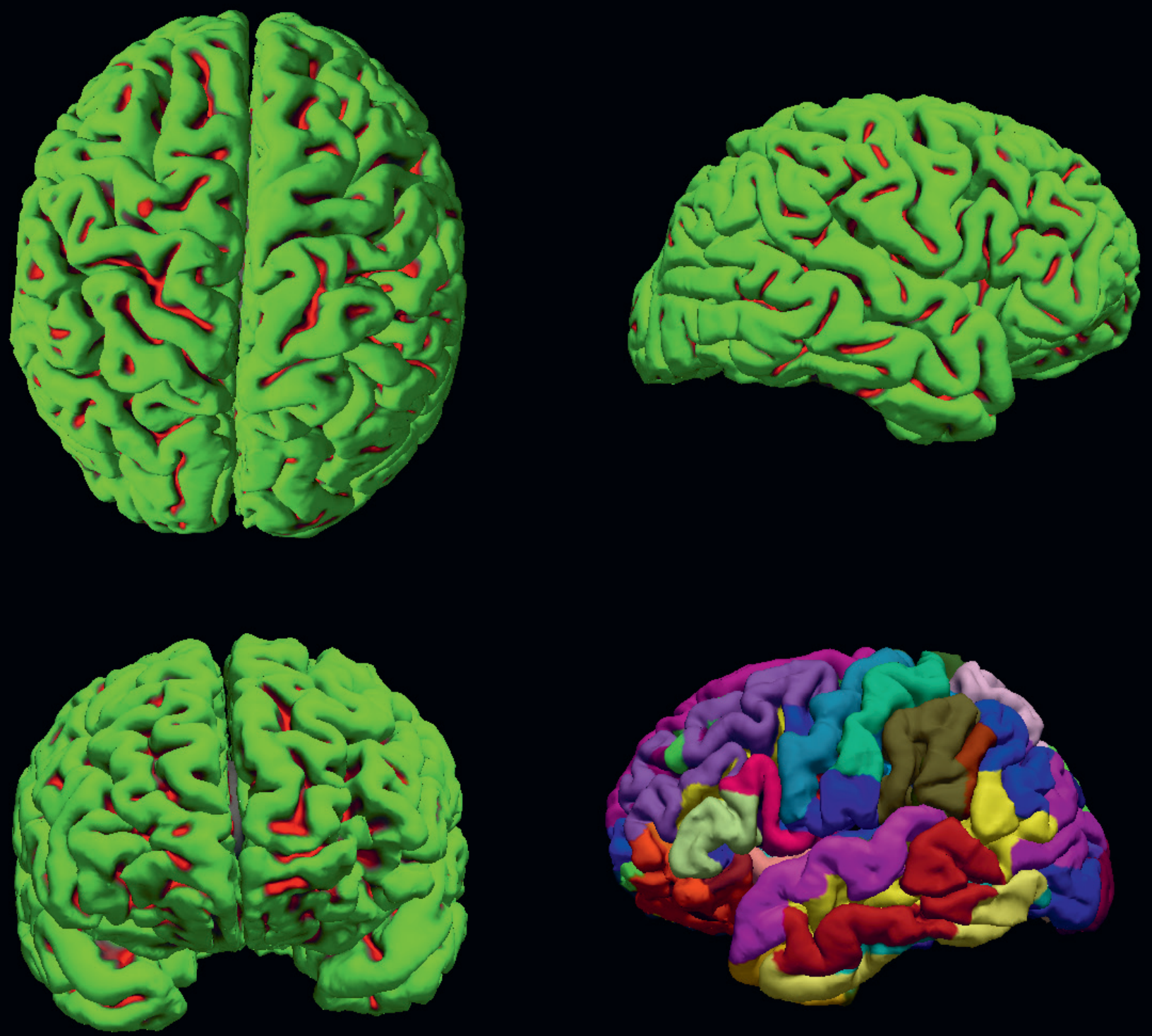

Fig. 5. Graphic representation of SIENAX field of view and standard space masking and whole brain segmentation

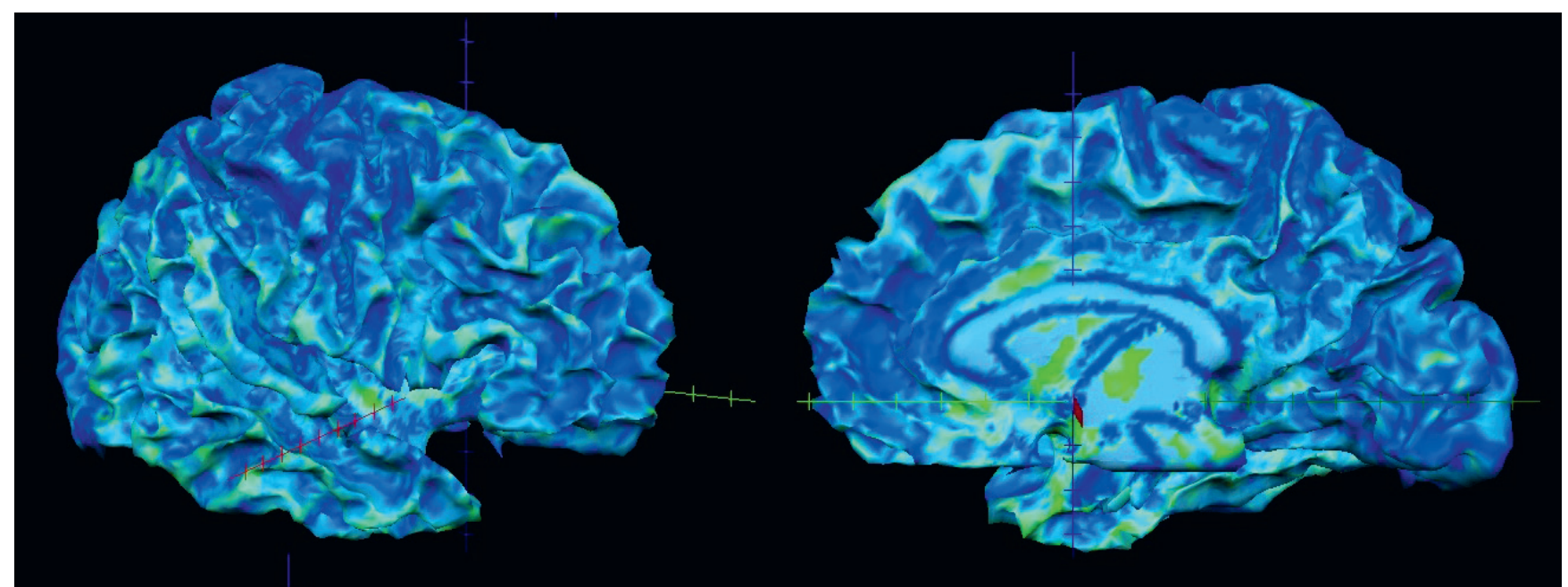

Fig. 6. 3D Pial surface (Freesurfer) 


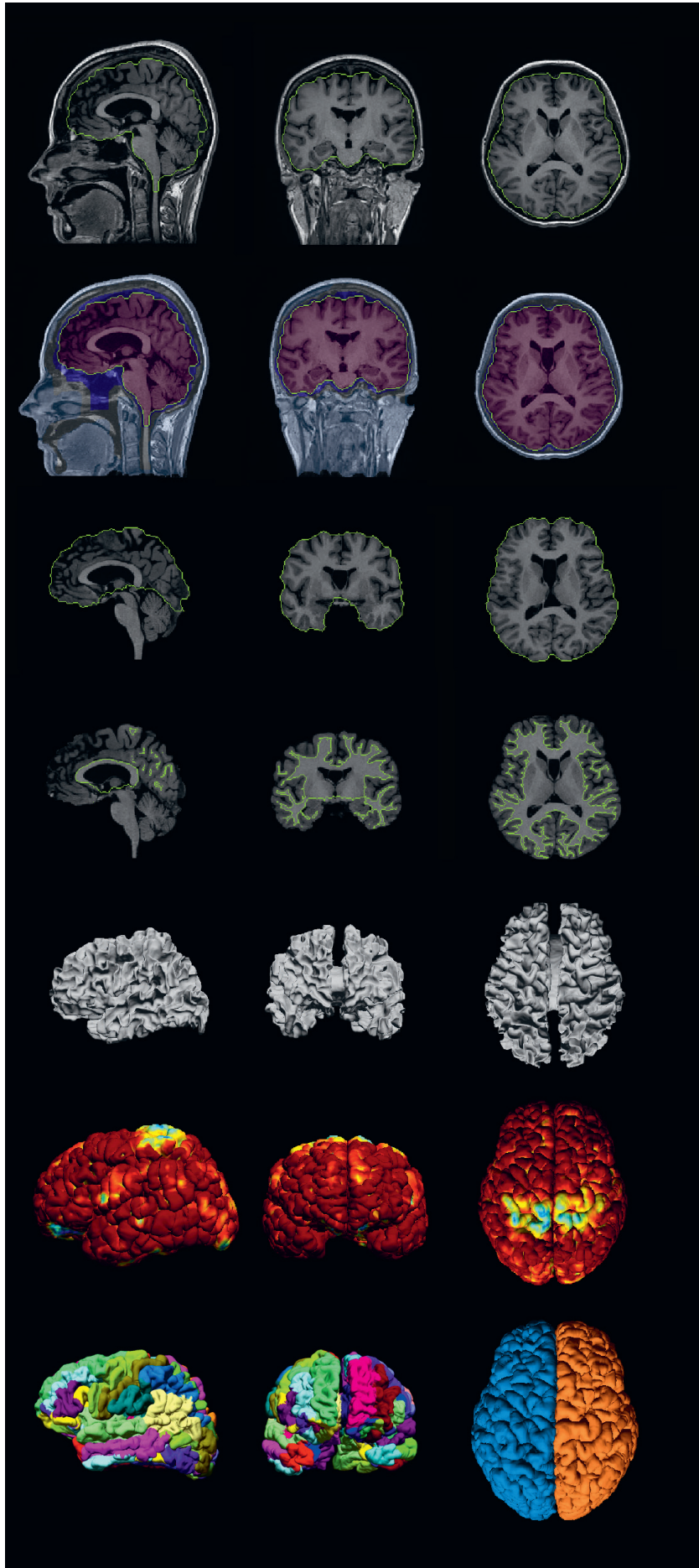

Fig. 7. WM/GM boundary surface reconstruction with overlaid cortical thickness map (BrainVoyager QX)

Most of this software, such as the FMRIB Software Library (FSL) package (FSL Analysis Group), BrainSuite (Ahmanson-Lovelace Brain Mapping Center/Biomedical Imaging Group, Los Angeles, USA) and FreeSurfer, are freely available and run online on a wide variety of hardware and software platforms (Fig. 5). ${ }^{65,66}$ That is why these automatic tools have been used in numerous studies. All of these programs can create 3D models of the most macroscopically visible structures in the human brain (Fig. 6).

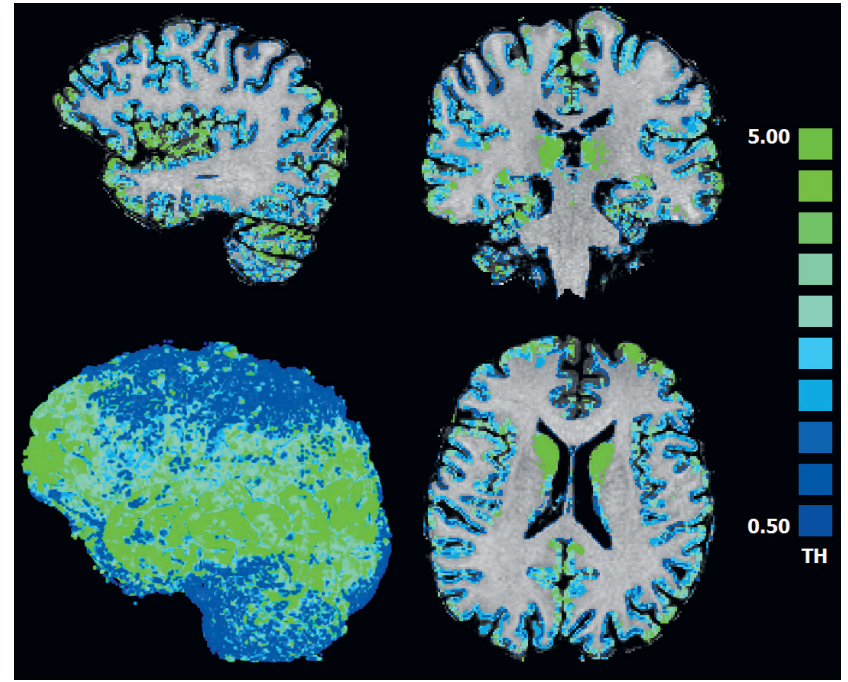

Fig. 8. Cortical Thickness Map - BrainVoyager

It is important to note that even when the voxel-based methods are used, due to the large variety of noise factors, the angulation of slices, the segmentation protocol, imaging protocol, patient-dependent factors, etc., there is a low probability of obtaining identical results of multiple studies performed during the same day on the same patient, even when using the same software. ${ }^{35,67}$ Despite these limitations, fully automated computational methods have shortened analysis time and allowed the assessment of large datasets. Due to advances in computational technology, the number of measurement errors is limited, thus the results are mostly reproducible and reliable. That is why they are extensively used in a number of clinical studies, especially for volumetric assessment of the whole brain and other intracranial structures such as lateral ventricles, WM, GM, and the hippocampus. ${ }^{2,68}$

Apart from its potential to help in the follow-up of many neurological diseases such as MS, this online software can aid researchers and clinicians in developing new treatments and monitoring their effectiveness.

\section{Conclusions}

Brain atrophy rate might be successfully used as an adjunctive biomarker of disease severity in the course of MS. A considerable amount of data derived from the latest studies confirms that measuring percentages of brain tissue loss over time is one of the best methods for quantifying neurodegeneration in MS and monitoring the progression of the disease.

Evaluating GM atrophy over the course of MS and its relationship to disability remains an important issue in the MS field. It should be stressed that GM atrophy could be an attractive potential marker of tissue loss, as it correlates better with clinical disability than other MRI measures and appears to be less sensitive to pseudoatrophy factors. 
New techniques need to be validated and MR protocols need to be standardized before they can be introduced into clinical practice. There is a need for a normative database, combined with important physiologic factors affecting estimations of brain atrophy. Obviously, efforts must be made to harness the potential of these measurements in assessing and monitoring pathologic evolution and treatment efficacy in MS.

\section{References}

1. Lassmann $\mathrm{H}$. Neuropathology in multiple sclerosis: New concepts. Mult Scler. 1998;4(3):93-98.

2. Bermel RA, Bakshi R. The measurement and clinical relevance of brain atrophy in multiple sclerosis. Lancet Neurol. 2006;5(2):158-170.

3. Lassmann H, Brück W, Lucchinetti C. Heterogeneity of multiple sclerosis pathogenesis: Implications for diagnosis and therapy. Trends Mol Med. 2001;7(3):115-121.

4. Ceccarelli A, Rocca MA, Pagani E, et al. A voxel-based morphometry study of grey matter loss in MS patients with different clinical phenotypes. Neuroimage. 2008;42(1):315-322.

5. Chetelat G, Baron J-C. Early diagnosis of Alzheimer's disease: Contribution of structural neuroimaging. Neuroimage. 2003;18(2):525-541.

6. Fox NC, Freeborough PA. Brain atrophy progression measured from registered serial MRI: Validation and application to Alzheimer's disease. J Magn Reson Imaging. 1997;7(6):1069-1075.

7. Smith SM, Zhang Y, Jenkinson M, et al. Accurate, robust, and automated longitudinal and cross-sectional brain change analysis. Neuroimage. 2002;17(1):479-489.

8. Rocca MA, Battaglini M, Benedict RHB, et al. Brain MRI atrophy quantification in MS: From methods to clinical application. Neurology. 2017;88(4):403-413.

9. Popescu V, Klaver R, Versteeg A, et al. Postmortem validation of MRI cortical volume measurements in MS. Hum Brain Mapp. 2016;37(6): 2223-2233.

10. Klaver R, Popescu V, Voorn P, et al. Neuronal and axonal loss in normal-appearing gray matter and subpial lesions in multiple sclerosis. J Neuropathol Exp Neurol. 2015;74(5):453-458.

11. Hardmeier M, Wagenpfeil S, Freitag P, et al; European IFN-1a in Relapsing MS Dose Comparison Trial Study Group. Rate of brain atrophy in relapsing MS decreases during treatment with IFNbeta-1a. Neurology. 2005;64(2):236-240.

12. Bakshi R, Thompson AJ, Rocca MA, et al. MRI in multiple sclerosis: Current status and future prospects. Lancet Neurol. 2008;7(7):615-625.

13. Fox RJ, Fisher E, Tkach J, Lee J-C, Cohen JA, Rudick RA. Brain atrophy and magnetization transfer ratio following methylprednisolone in multiple sclerosis: Short-term changes and long-term implications. Mult Scler. 2005;11(2):140-145.

14. Ceccarelli A, Rocca MA, Pagani E, et al. A voxel-based morphometry study of grey matter loss in MS patients with different clinical phenotypes. Neuroimage. 2008;42(1):315-322.

15. Kutzelnigg A, Lucchinetti CF, Stadelmann C, et al. Cortical demyelination and diffuse white matter injury in multiple sclerosis. Brain. 2005;128(Pt 11):2705-2712.

16. Pirko I, Johnson AJ, Chen Y, et al. Brain atrophy correlates with functional outcome in a murine model of multiple sclerosis. Neuroimage. 2011;54(2):802-806.

17. De Stefano N, Narayanan S, Francis GS, et al. Evidence of axonal damage in the early stages of multiple sclerosis and its relevance to disability. Arch Neurol. 2001;58(1):65-70.

18. Morgen K, Sammer G, Courtney SM, et al. Evidence for a direct association between cortical atrophy and cognitive impairment in relapsing-remitting MS. Neuroimage. 2006;30(3):891-898.

19. Ellwardt E, Zipp F. Molecular mechanisms linking neuroinflammation and neurodegeneration in MS. Exp Neurol. 2014;262(Pt A):8-17.

20. Zindler E, Zipp F. Neuronal injury in chronic CNS inflammation. Best Pract Res Clin Anaesthesiol. 2010;24(4):551-562.

21. De Stefano N, Iannucci G, Sormani MP, et al. MR correlates of cerebral atrophy in patients with multiple sclerosis. J Neurol. 2002;249(8):1072-1077.
22. Gonçalves LI, Dos Passos GR, Conzatti LP, et al. Correlation between the corpus callosum index and brain atrophy, lesion load, and cognitive dysfunction in multiple sclerosis. Mult Scler Relat Disord. 2018;20: 154-158.

23. Wiggermann V, Ibs I, Schoerner S, et al. Exploring mechanisms of multiple sclerosis lesion evolution using advanced MRI. Neurology. 2016;86(Suppl 16):I10.012.

24. Zivadinov R, Jakimovski D, Gandhi S, et al. Clinical relevance of brain atrophy assessment in multiple sclerosis: Implications for its use in a clinical routine. Expert Rev Neurother. 2016;16(7):777-793.

25. Ohara N, Suzuki H, Suzuki A, et al. Reversible brain atrophy and cognitive impairment in an adolescent Japanese patient with primary adrenal Cushing's syndrome. Neuropsychiatr Dis Treat. 2014;10:1763-1767.

26. Zivadinov R, Bergsland N, Dolezal O, et al. Evolution of cortical and thalamus atrophy and disability progression in early relapsing-remitting MS during 5 years. AJNR Am J Neuroradiol. 2013;34:1931-1939.

27. Duning T, Kloska S, Steinsträter $\mathrm{O}$, Kugel H, Heindel W, Knecht S. Dehydration confounds the assessment of brain atrophy. Neurology. 2005;64(3):548-550.

28. Meyers SM, Tam R, Lee JS, et al. Does hydration status affect MRI measures of brain volume or water content? J Magn Reson Imaging. 2016;44(2):296-304.

29. Mellanby AR, Reveley MA. Effects of acute dehydration on computerized tomographic assessment of cerebral density and ventricular volume. Lancet. 1982;2(8303):874.

30. Heinz ER, Martinez J, Haenggeli A. Reversibility of cerebral atrophy in anorexia nervosa and Cushing's syndrome. J Comput Assist Tomogr. 1977;1(4):415-418.

31. Addolorato G, Taranto C, De Rossi G, Gasbarrini G. Neuroimaging of cerebral and cerebellar atrophy in anorexia nervosa. Psychiatry Res. 1997;76(2-3):139-141.

32. Tomassini V, d'Ambrosio A, Petsas N, et al. The effect of inflammation and its reduction on brain plasticity in multiple sclerosis: MRI evidence. Hum Brain Mapp. 2016;37(7):2431-2445.

33. Rocca MA, Pagani E, Ghezzi A, et al. Functional cortical changes in patients with multiple sclerosis and nonspecific findings on conventional magnetic resonance imaging scans of the brain. Neuroimage. 2003;19(3):826-836.

34. Rocca MA, Mezzapesa DM, Falini A, et al. Evidence for axonal pathology and adaptive cortical reorganization in patients at presentation with clinically isolated syndromes suggestive of multiple sclerosis. Neuroimage. 2003;18(4):847-855.

35. Giorgio A, Battaglini M, Smith SM, De Stefano N. Brain atrophy assessment in multiple sclerosis: Importance and limitations. Neuroimaging Clin N Am. 2008;18(4):675-686,xi.

36. Chen JT, Collins DL, Atkins HL, Freedman MS, Galal A, Arnold DL; Canadian MS BMT Study Group. Brain atrophy after immunoablation and stem cell transplantation in multiple sclerosis. Neurology. 2006;66(12):1935-1937.

37. De Stefano N, Arnold DL. Towards a better understanding of pseudoatrophy in the brain of multiple sclerosis patients. Mult Scler. 2015;21(6):675-676.

38. Zivadinov R. Steroids and brain atrophy in multiple sclerosis. J Neurol Sci. 2005;233(1-2):73-81.

39. Cohen JA, Barkhof F, Comi G, et al; TRANSFORMS Study Group. Oral fingolimod or intramuscular interferon for relapsing multiple sclerosis. N Engl J Med. 2010;362(5):402-415.

40. Vidal-Jordana A, Sastre-Garriga J, Pérez-Miralles F, et al. Brain volume loss during the first year of interferon-beta treatment in multiple sclerosis: Baseline inflammation and regional brain volume dynamics. J Neuroimaging. 2016;26(5):532-538.

41. Gordon N. Apparent cerebral atrophy in patients on treatment with steroids. Dev Med Child Neurol. 1980;22(4):502-506.

42. Lyen KR, Holland IM, Lyen YC. Reversible cerebral atrophy in infantile spasms caused by corticotrophin. Lancet. 1979;2(8132):37-38.

43. Filippi $M$, Rovaris $M$, Inglese $M$, et al. Interferon beta-1a for brain tissue loss in patients at presentation with syndromes suggestive of multiple sclerosis: A randomised, double-blind, placebo-controlled trial. Lancet. 2004;364(9444):1489-1496.

44. Prins M, Schul E, Geurts J, van der Valk P, Drukarch B, van Dam A-M. Pathological differences between white and grey matter multiple sclerosis lesions. Ann N Y Acad Sci. 2015;1351:99-113. 
45. Calabrese M, Agosta F, Rinaldi F, et al. Cortical lesions and atrophy associated with cognitive impairment in relapsing-remitting multiple sclerosis. Arch Neurol. 2009;66(9):1144-1150.

46. Diker S, Has AC, Kurne A, Göçmen R, Oğuz KK, Karabudak R. The association of cognitive impairment with gray matter atrophy and cortical lesion load in clinically isolated syndrome. Mult Scler Relat Disord. 2016;10:14-21.

47. Paul F. Pathology and MRI: Exploring cognitive impairment in MS. Acta Neurol Scand. 2016;134(Suppl 200):24-33. doi:10.1111/ane.12649

48. Cappellani R, Bergsland N, Weinstock-Guttman B, et al. Subcortical deep gray matter pathology in patients with multiple sclerosis is associated with white matter lesion burden and atrophy but not with cortical atrophy: A diffusion tensor MRI study. AJNR Am J Neuroradiol. 2014:35(5):912-919.

49. Datta S, Staewen TD, Cofield SS, et al; MRI Analysis Center at Houston; CombiRx Investigators Group. Regional gray matter atrophy in relapsing remitting multiple sclerosis: Baseline analysis of multicenter data. Mult Scler Relat Disord. 2015;4(2):124-136.

50. Sanfilipo MP, Benedict RHB, Weinstock-Guttman B, Bakshi R. Gray and white matter brain atrophy and neuropsychological impairment in multiple sclerosis. Neurology. 2006;66(5):685-692.

51. Henry RG, Shieh M, Okuda DT, Evangelista A, Gorno-Tempini ML, Pelletier D. Regional grey matter atrophy in clinically isolated syndromes at presentation. J Neurol Neurosurg Psychiatry. 2008;79(11):1236-1244.

52. Azevedo CJ, Overton E, Khadka S, et al. Early CNS neurodegeneration in radiologically isolated syndrome. Neurol Neuroimmunol Neuroinflamm. 2015;2(3):e102.

53. Dalton CM, Chard DT, Davies GR, et al. Early development of multiple sclerosis is associated with progressive grey matter atrophy in patients presenting with clinically isolated syndromes. Brain. 2004; 127(Pt 5):1101-1107.

54. Kincses ZT, Tóth E, Bankó N, et al. Grey matter atrophy in patients suffering from multiple sclerosis. Ideggyogy Sz. 2014;67:293-300.

55. Steenwijk MD, Geurts JJG, Daams M, et al. Cortical atrophy patterns in multiple sclerosis are non-random and clinically relevant. Brain. 2016;139(Pt 1):115-126.
56. Filippi M, Rocca MA.MRI evidence for multiple sclerosis as a diffuse disease of the central nervous system. J Neurol. 2005;252(Suppl):v16-24.

57. Calabrese M, Poretto V, Favaretto A, et al. Cortical lesion load associates with progression of disability in multiple sclerosis. Brain. 2012; 135(Pt 10):2952-2961.

58. Chataway J. When confronted by a patient with the radiologically isolated syndrome. Pract Neurol. 2010;10(5):271-277.

59. Hasan KM, Walimuni IS, Abid H, et al. Multimodal quantitative magnetic resonance imaging of thalamic development and aging across the human lifespan: Implications to neurodegeneration in multiple sclerosis. J Neurosci. 2011;31(46):16826-16832.

60. Giorgio A, De Stefano N. Clinical use of brain volumetry. J Magn Reson Imaging. 2013;37(1):1-14.

61. Næss-Schmidt E, Tietze A, Blicher JU, et al. Automatic thalamus and hippocampus segmentation from MP2RAGE: Comparison of publicly available methods and implications for DTI quantification. Int J Comput Assist Radiol Surg. 2016;11(11):1979-1991.

62. Manjón J V, Coupé P. volBrain: An online MRI brain volumetry system. Front Neuroinform. 2016;10:30.

63. Hao Y, Wang T, Zhang X, et al. Local label learning (LLL) for subcortical structure segmentation: Application to hippocampus segmentation. Hum Brain Mapp. 2014;35(6):2674-2697.

64. Yeo BTT, Sabuncu MR, Desikan R, Fischl B, Golland P. Effects of registration regularization and atlas sharpness on segmentation accuracy. Med Image Anal. 2008;12(5):603-615.

65. Fischl B. FreeSurfer. Neuroimage. 2012;62(2):774-781.

66. Shattuck DW, Prasad G, Mirza M, Narr KL, Toga AW. Online resource for validation of brain segmentation methods. Neuroimage. 2009; 45(2):431-439.

67. Collins DL, Pruessner JC. Towards accurate, automatic segmentation of the hippocampus and amygdala from MRI by augmenting ANIMAL with a template library and label fusion. Neuroimage. 2010;52(4): 1355-1366.

68. Jenkinson M, Beckmann CF, Behrens TEJ, Woolrich MW, Smith SM. FSL. Neuroimage. 2012;62(2):782-790. 